\title{
Parent Training Through Play: Parent-Child Interaction Therapy With a Hyperactive Child
}

\author{
Brian D. Johnson \\ Leslie C. Franklin \\ Kathryn Hall \\ University of Northern Colorado \\ Loreto R. Prieto \\ University of Oklahoma
}

A ttention deficit hyperactivity disorder (ADHD) is the most common childhood behavior disorder. Characterized by developmentally inappropriate levels of inattention, impulsivity, and/or hyperactivity, it affects $5 \%$ to $7 \%$ of the population. Barkley (1998) noted that by its nature, children with ADHD are at increased risk to experience relationship conflicts, particularly when environmental demands exceed their capacity to cope. As a result, the entire family system of ADHD children experiences significantly more stress and conflict when compared with controls. Their parents tend to adopt more negative parenting styles (Cunningham \& Barkley, 1979), view their abilities to parent less positively (Mash \& Johnston, 1990), and are at increased risk for experiencing depression, anxiety, and marital discord (Lahey et al., 1988). Children with ADHD are rated as having significantly more symptoms of anxiety, depression, low self-esteem, learning difficulties, and behavior problems than controls (Barkley, 1998). In addition, they also experience significantly more conflicts with their peers and siblings (Mash \& Johnston, 1990). Children with ADHD are frequently disliked by their peers due to the amount of adult attention required and conflict created (Dixon, 1995).

Parenting programs have become popular treatment approaches to help families cope with ADHD children (Barkley, 1998). Most parenting programs propose that when children have ADHD, typical parenting approaches are not as effective. Consequently, parents are taught about the importance of consistency, follow-through, and using behavior management techniques. These programs also frequently discuss the importance of spending quality time with children, having parents choose their battles and dealing with school-related concerns. Most programs discuss these topics

Authors' Note: Requests for reprints of this manuscript should be sent to Dr. Brian D. Johnson, Division of Professional Psychology, University of Northern Colorado, Greeley, CO 80639. in classroom style workshops where a group of parents assemble and learn what to do from a counselor. Parents are given homework assignments to apply what they have learned and the counselor will problem-solve any difficulties the parents encounter.

Few parenting programs address family issues by having parents and children interact with one another during sessions. One program that does is Parent-Child Interaction Therapy (PCIT) (Hembree-Kigin \& McNeil, 1995). Originally developed by Shelia Eyberg as a therapy for families with behaviorally disturbed children, PCIT has been shown to be effective at counseling children with problems ranging from ADHD, separation anxiety, depression, self-injurious behavior, post-divorce adjustment, and abuse (HembreeKigin \& McNeil, 1995).

In this article, we describe how PCIT was used to counsel a family that had a 7-year-old boy with ADHD who was beginning to exhibit oppositional-defiant behaviors. According to Barkley (1998), approximately 50\% of children with ADHD develop oppositional-defiant disorder (ODD) and approximately $50 \%$ of this group eventually develop the more serious conduct disorder (CD). Studies have shown that early intervention helps to prevent the progression of these more serious conditions (McGee, Partidge, Williams, \& Silva, 1991). PCIT is best suited for treating children between the ages of 3 and 9 years old.

Although many family interventions attempt to improve parent-child relationships, PCIT attempts to do this through teaching parents some fundamental relationship-building techniques employed by play therapists. Techniques such as describing what a child is doing, reflecting appropriate speech and feelings, and praising appropriate behaviors are taught to parents. Parents practice these new skills during special playtimes both within the session and at home. These therapeutic skills frequently generalize to situations outside of the special playtime, and a reduction in family stress is 
often reported. Once an improvement in the parent-child relationship is noted, PCIT then begins to offer parents strategies to deal with the behavioral problems commonly associated with ADHD.

In this case study, we have attempted to emphasize specific techniques used to improve the parent-child relationship. We also described the types of recommendations made to help the parents deal with their son's acting-out behaviors. Barkley (1998) summarized a series of studies that suggested familial stress is the result of an ADHD child's acting-out behavior. Consequently, giving parents effective techniques to deal with behavior problems reduces family stress and can further enhance relationships among all family members. The interested reader is encouraged to consult the Hembree-Kigin and McNeil (1995) book Parent-Child Interaction Therapy for a more detailed discussion of this approach.

\section{CASE}

Joey, a 7-year-old first grader, was referred by his pediatrician for an evaluation and treatment of problems associated with ADHD. Joey had been diagnosed with ADHD at the age of 6 and had been taking Ritalin for approximately 7 months. His parents reported that he was an only child and had always been "difficult" in that he had colic as an infant, was very "independent" as a toddler and active throughout his development. They had hoped that Joey would eventually "outgrow" these difficulties, but when his teachers also expressed concerns, they sought assistance from a pediatrician. They reported that although the Ritalin seemed to have improved Joey's inattention and restlessness, he had become increasingly more noncompliant, argumentative, and aggressive. When told to do something, Joey would either actively refuse to do so or do the opposite of what he had been told. His mother tearfully described a recent incident at the supermarket where Joey had run away from her, opened up a bag of potato chips and emptied its contents onto the floor before she could reach him. Another customer whispered, "I hope you're going to do something to make sure he doesn't pull that stunt again." Feeling embarrassed, angry and dejected, she carried a wailing Joey out of the store, leaving her half full cart in the aisle.

This was not the first time that they had been given "advice" about how to manage Joey's behavior from family members, friends, and strangers. Although his parents had tried a number of different discipline strategies, they reported to us "nothing seems to work." With their parental self-esteem at an all-time low, Joey's parents expressed a desire to learn new strategies for managing his difficult behaviors. Given their history of frustration and embarrassment with Joey, we were prepared for them to be somewhat resistant to many of our recommendations, as they would have likely already tried many of them. As a way to work around this, we acknowledged that typical parenting strategies are not effective for children with ADHD. If they were effective, all the free advice they had been receiving would have worked. We then said that we would be showing them some new techniques and some new ways to implement old techniques. We informed them that these techniques are frequently only used by therapists and counselors. It has been our experience that with this type of explanation, most parents are much more eager to try our recommendations even if we are recommending something that did not work for them in the past. In addition, parents usually become much more hopeful about their situations.

\section{Intake Session}

There were several important topics discussed during the intake. First, we allowed Joey and his parents to describe their concerns. It was also important for us to clarify any ambiguity regarding their concerns. Second, we obtained specific descriptions of Joey's behavior and how his parents reacted to them. It has been our experience that parents hold back when discussing such issues in front of their young children, so we encouraged Joey to go to an adjoining playroom during this part of the intake. As his parents described how they dealt with things, a number of problems became apparent. For example, when Joey refused to cooperate to a parental request, his parents would "try to explain to him why it was important to do what he had been told." As they were to learn later, these explanations were actually making the situation worse by giving attention to an attention-seeking behavior. In addition, while his parents indeed tried many forms of discipline with little success (e.g., spanking, removal of privileges, time-outs), it became apparent that they were not carrying out their discipline efforts in a consistent manner. Furthermore, they were focusing almost exclusively on behaviors that they wanted stopped and not on the behaviors that they wanted increased. By helping his parents to recognize how their own reactions escalated Joey's uncooperative behaviors, we were able to give them a greater sense of control in managing problem situations.

A third topic discussed was to evaluate the impact of Joey's behavior on the family. Given that Joey was an only child, our discussion focused on each parent's relationship with Joey and their marital relationship. Both parents admitted that much of the time they did not enjoy being Joey's parents and they felt guilty for having these feelings. They also admitted that their marital relationship had suffered as a result of regular conflicts surrounding ways to deal with Joey. At this point, we assessed how committed each parent was to their marriage. If there had been any question about their commitment, we would have recommended concurrent marital therapy.

We also assessed Joey's compliance to parental requests by asking, "During the course of the day, if you asked Joey to do 10 different things, how many of them would he do the first time that you asked him?" Most parents with ADHD and/or 
ODD children respond with zero to one times. Joey's mother said "zero" and his father said "three, with a lot of back talk." We then provided them with a frame of reference by explaining that children his age can be expected to cooperate $50 \%$ to $70 \%$ of the time. At that time, we knew increasing Joey's cooperation to parental requests would be a major emphasis during therapy.

Finally, we asked Joey's parents to describe how they were disciplined as children. Like most parents, Joey's admitted that when they were stressed, they often "lose it" and exhibit parenting behaviors that their parents exhibited when they were young. Given that neither of his parents wanted to parent Joey exactly the way they were, they often felt "angry" and "guilty" when they lost it with Joey. By discussing the relationship of stress and the escalation of family conflict, we were able to begin a dialogue to encourage Joey's parents to practice some stress management techniques such as relaxation training and parental time-outs.

At the end of the intake, Joey's parents completed two questionnaires that allowed us to further determine the nature and extent of his behavior problems. The first questionnaire was the Child Behavior Checklist (CBCL) (Achenbach \& Endelbrock, 1983) and the second was the Parent Stress Inventory (PSI) (Abidin, 1995). The CBCL allowed us to evaluate for both internalizing (e.g., withdrawal, anxiety/depression) and externalizing (e.g., aggression, hyperactive) behaviors. We were also able to evaluate how consistent the parents were in their perceptions of Joey's behaviors. The PSI collected information regarding how Joey's parents perceived his behavior (e.g., demanding, reinforcing to parent, moody). It also obtained information regarding how his parents perceived themselves (e.g., parenting competence, attachment to child, depression).

\section{Initial Treatment Session}

The initial treatment session started with Joey's parents receiving feedback regarding their responses to the questionnaires. Most parents appreciate learning how their ratings compared with those of other parents. On the CBCL, Joey's parents endorsed significant concerns related to inattention, aggression, and hyperactive behaviors. Their consistency was within the above-average range, suggesting that not only were they both endorsing similar behavioral concerns but also that they agreed on the frequency to which those behaviors occurred. On the PSI, both parents described Joey as hyperactive, having difficulty adjusting to change, and being moody. His mother endorsed moderately high levels of stress related to her perceived lack of competence in parenting, lack of support from her spouse, and feelings of isolation. Joey's father also endorsed concerns related to his perceived lack of competence in parenting. Using this information, we were able to focus our interventions on specific concerns expressed by Joey's parents. For example, both were concerned about their competence to parent, so we increased the amount of time they spent practicing new parenting behaviors.

We also conducted a systematic observation of Joey interacting with one of his parents. Because this observation is usually only done with one parent, we let his parents decide who would go. Joey's mother volunteered because she was having more difficulties with him. For the observation, we had Joey and his mom go into a playroom where there was a table and two chairs seated side by side. On the table were various toys that encouraged constructive or creative play (e.g., Legos, farm animals, markers, and paper). Through a one-way mirror, we observed Joey and his mother playing at the table for 15 minutes. During the first 5 minutes, Joey's mother was instructed to let him direct the play and she was to follow his lead. The second 5 minutes involved her getting Joey to play with a different toy and to have him follow her lead. The final 5 minutes involved her getting Joey to pick up the toys. These observations were coded using the Dyadic Parent-Child Interaction Coding (DPIC) system (Eyberg \& Robinson, 1983). With the DPIC, we were able to code the frequency of various parent (e.g., types of commands, types of praise, responses to misbehavior) and child (e.g., compliant, noncompliant, disruptive) behaviors. Joey's compliance to his mother's requests were $75 \%$ when he was directing the interaction. When he was following her lead, however, his compliance decreased to $20 \%$ and it fell to $5 \%$ during cleanup. Joey's mother frequently gave attention to uncooperative and disruptive behaviors, and her commands were frequently indirect. During the entire 15 minute interaction, only two instances of praise were noted.

Following the behavioral observations, Joey remained in the playroom while we met with his parents in an adjoining office. With his parents, we discussed the first phase of PCIT, which included the rationale for doing play therapy, the do's and don'ts of play therapy, the types of toys to use during play therapy, and how to structure the therapy in their home. The rationale given for doing PCIT was that because children with ADHD are "wired differently," specialized parenting strategies are often necessary. With PCIT, parents learn strategies to help their ADHD children better cope with their difficulties. Topics related to consistency, praise for appropriate behavior, and normal child development were discussed.

Joey's parents were told that they would be doing play therapy because young children often learn best when playing. In addition, the therapy provides an opportunity for regularly scheduled positive parent-child interactions. The do's of play therapy were to describe what Joey was doing with the toys, to reflect back what he said or how he appeared to be feeling, to imitate what he was doing, and to praise appropriate behavior. The don'ts of play therapy included avoiding criticisms, commands, and questions. The parents were also encouraged to ignore obnoxious or whining behaviors.

Types of toys that we recommended included toys that encouraged creative play (e.g., Legos, markers, farm animal 
set). Toys that encouraged rough or aggressive play (e.g., action figures, guns, bats) were to be avoided. In addition, games that have defined rules or promote competition (e.g., Uno, Sorry) were also to be avoided.

Finally, ways to structure play therapy sessions within their home were discussed. The parents were told to do special playtime at a table that was removed from other types of distractions. They were encouraged to individually engage in special playtime with Joey for 5 minutes everyday. Although it was unlikely that both parents would actually do play therapy everyday, it was our hope that he would get it from at least one parent on a daily basis. We also encouraged them to limit themselves to 5 minutes every day. Although 5 minutes may not seem like much time, Hembree-Kigin and McNeil (1995) reported that parents are more likely to do play therapy on a consistent basis if it is limited to 5 minutes a day.

\section{Child-Directed Interaction Phase}

During this phase of PCIT, improving the parent-child relationship through the play therapy was emphasized. Typically, this phase lasts from three to five sessions. Joey and one parent would go into the play therapy room, and the other parent and therapists would observe the interaction through a one-way mirror. Halfway through the session, the parents would switch places so Joey had an opportunity to interact with each parent. A "bug in the ear" device was worn by the parent interacting with Joey so therapists could coach him or her. Initially, his parents were given specific suggestions regarding the do's and don'ts of play therapy (e.g., "Since Joey is drawing, why don't you go ahead and draw something too"). They were also given explicit examples of things to say and initially Joey's parents would frequently repeat back statements suggested by a therapist (e.g., "It is so much fun sitting at the table and playing with you"). As his parents became more confident in their play therapy skills, less time was spent coaching them on what to say and do.

Coaching then emphasized the positive aspects of the parent-child relationship (e.g., "See how much his face lights up when you tell him that you like his ideas"). Attempts were also made to challenge some of the assumptions his parents had made about Joey (e.g., "While it may look like Joey is ignoring you on purpose, I am not sure he understood what you said, try telling him again"). Given that neither parent felt competent in his or her ability to manage Joey's behavior, much time was devoted to recognizing examples of their competence (e.g., "You did a great job of ignoring his uncooperative behavior and getting him back to the table"). We also helped his parents to recognize trigger events that led to uncooperative behavior (e.g., "You started to raise your voice just before he began pushing the Legos off the table. I wonder if he is more likely to get set off when you raise your voice?").

Toward the end of the child-directed interaction (CDI) phase, we often observe examples of the play therapy "talk" generalizing to situations outside of playtime, for example, in the waiting room. It is also not uncommon for parents to start receiving praise from their child (e.g., "That's a great idea mom"). It has been our experience that many children with ADHD have a knee-jerk reaction to be uncooperative to see how others will respond to them. The play therapy seems to help reduce the intensity of the knee-jerk and an increase in cooperation is often reported. Although improvement in overall behavior is often seen, play therapy alone is seldom sufficient to significantly reduce the frequency of behavior problems encountered by parents of an ADHD child. The second phase of PCIT involved a discipline component that directly addressed these specific behavior problems.

Although many parents are eager to initiate the discipline component of PCIT, we believe that time first must be spent emphasizing activities that improve the parent-child relationship. We have found that when we hurry to discipline, we are much more likely to encounter parent-child power struggles, and we believe the overall effectiveness of the program is diminished. The counselor must constantly weigh the needs of the family with the importance of improving the relationship. Frequently, parents need to be encouraged to be patient and recognize that their child's problem behaviors did not develop overnight and that they will not diminish that quickly either.

\section{Parent-Directed Interaction Phase}

Following the establishment of the play therapy and the ensuing improvement in the parent-child relationship, the second phase of PCIT is initiated. The parent-directed interaction (PDI) phase involved teaching Joey's parents some specific behavioral techniques to manage his disruptive behaviors. PDI typically takes three to five sessions, but depending on the severity of the family's difficulties, it can take considerably longer. We devoted five sessions to PDI with Joey and his parents. Although PDI may appear to be mechanistic and behavioral on the surface, in reality, more time is spent to further strengthen the parent-child relationship and enhance parent-child self-esteem than on discipline.

The first PDI session involved meeting only with Joey's parents. At this meeting, we discussed the family's progress to date and there was an opportunity for his parents to share stories of their perceived accomplishments. During this session, we encouraged Joey's parents to meet four preconditions before they ever discipline him. The preconditions were that (a) Joey knew exactly what he was supposed to do (or not do), (b) Joey knew what the consequence would be if he did not (or continued to do) the behavior, (c) Joey was developmentally capable of doing what he was being asked to do, and (d) his parents' own frustration and anger were under control before they carried out the discipline.

We also taught his parents the importance of recognizing different categories of behavior problems. For example, about $60 \%$ of behavior management problems in children result from failure to comply to parental requests (Hembree- 
Kigin \& McNeil, 1995). Consequently, the PDI phase of therapy initially focused on improving Joey's compliance and cooperation. We achieved this by incorporating "minding exercises" into the special playtime. Periodically, Joey's parents were instructed to say, "Now we are going to practice minding. Please put the red Lego into my hand." Joey's parents simultaneously pointed to the red Lego and to their open hand. By doing this, we were able to ensure that the first and third preconditions were met (i.e., Joey knew what was expected of him and he was developmentally capable of doing it). If Joey cooperated, his parents would immediately say something like, "Joey, thank you for minding so quickly. I really like it when you do that and now we can stay at the table and play." Once Joey regularly complied to small requests, more real-world requests were incorporated into playtime (e.g., "Joey, please take off your shoes and put them by the door").

We also taught his parents how to make their requests in a manner that increased the likelihood Joey would cooperate. For example, they gave direct versus indirect commands, gave one-step versus multistep commands, were polite and respectful, and gave commands only when necessary. Much time was spent on helping his parents to choose their battles and recognizing that although Joey may be developmentally capable of putting his shoes away when he was well rested, he might not be capable of doing so when very tired. Consequently, we emphasized having his parents monitor for situations that trigger acting-out behaviors.

Throughout the PDI phase, Joey's parents continued having special playtime at home. We then gradually introduced minding exercises into home playtime. Once Joey was minding well during playtime at home, his parents started to give him minding exercises outside of playtime. This helped to facilitate the generalization of learning to situations outside of our clinic and the play therapy environment. Generalization was further enhanced by giving Joey minding exercises away from home as well (e.g., at grandparents' home, in stores).

Obviously, Joey would not be cooperative all the time and in all environments, so his parents needed a consistent strategy to deal with uncooperative behaviors. During playtime, if Joey did not comply to a minding exercise such as "please put the red Lego into my hand," his parents were instructed to show Joey two fingers and say, "Joey, you have two choices. You can put the red Lego into my hand or you can go to time-out." By doing this, his parents were meeting the second precondition for discipline (Joey knows exactly what the consequence will be). If Joey cooperated, he was praised for minding and he and his parent continued with special playtime. If he refused to cooperate, he was immediately taken from the table and put into a time-out chair a few steps away. The time-out chair was kept close to the play therapy table because children with ADHD appear to learn best when the consequences of their actions are immediate. In addition, having the chair close decreased the likelihood that the situation would further escalate if Joey started hitting or kicking as he was being carried. Immediate consequences also helped to ensure that the fourth precondition for discipline had been met (Joey's parents own anger was under control).

Although types of consequences vary from family to family, we like to use the time-out chair because it can be used immediately and it is portable. In addition, when done correctly time-out is an extremely effective intervention. Unfortunately, time-out is seldom done correctly as children continue to receive parental attention when they are in time-out. We instructed Joey's parents on appropriate ways to implement time-out and suggested a useful technique that we call "talking to the walls." When talking to the walls, his parents talked as if Joey was not in the room. They were instructed to say what Joey needed to do to receive their attention, rather than giving attention to his inappropriate behavior. For example, when Joey was in time-out and yelling, his father was instructed to engage in the following dialogue to the walls: "I cannot talk to Joey until he sits quietly in the time-out chair. Joey needs to sit quietly, then I can talk to him. I sure hope Joey will sit quietly soon so we can talk. He cannot get out of the time-out chair until he sits quietly and I would like to play with him ... oh, thank you Joey for sitting quietly."

One of the most common reasons parents give us for why time-out does not work is that the child will not stay in the time-out chair. Joey's parents had tried time-out by putting Joey in his room. When he would not stay there, they would hold his door shut. Often, a tug-of-war would ensue with the bedroom door being pushed open and closed. Eventually, his parents would either let him out of the room or the situation would escalate into Joey receiving a spanking. We helped his parents to recognize that when they are talking to Joey and trying to prevent him from leaving his room, he is not really in time-out. In fact, this situation can be quite stimulating for a young, uncooperative child. Unfortunately, when his parents gave up and let him out, they encouraged similar struggles in the future. When things escalated to physical punishment, everyone in the family was left feeling angry and guilty.

Consequently, we needed to help his parents develop a "Plan B" strategy for when Joey would not go to time-out. The Plan B that we recommended was a therapeutic "holding chair." Many times, parents will try to hold an uncooperative child in their lap, however, this can result in someone getting hurt. To decrease this possibility, the holding chair was a separate chair in which Joey sat. His parents then crossed his arms and held him around the wrists using only their thumbs and forefingers. They would either stand or kneel behind the chair so there would be no additional physical contact. Joey was then held in this position for 30 seconds and immediately put back into the original time-out chair. The holding chair can be effective for young children and Joey needed to go to the holding chair two times before he regularly stayed in the time-out chair. Other types of Plan B consequences can 
include losing privileges, isolation to another room, and a two-swat spank (Hembree-Kigin \& McNeil, 1995).

Once we had successfully increased minding, we then focused on a second class of behavior problems that we referred to as "stop behaviors." Stop behaviors represented any behavior that Joey already did and his parents wanted to make sure would not happen again (e.g., running away in the grocery store and opening up a bag of chips). Other examples of common stop behaviors include hitting, spitting, swearing, or fire-setting. We addressed this class of behavior problems by setting up house rules. A third class of behavior problems that we addressed were public behaviors. Public behaviors reflected how he behaved in various public places and were addressed by establishing public rules. If Joey violated a house or public behavior, he went to time-out.

Throughout this process, Joey's parents praised his appropriate behaviors and used time-out only when necessary. They recognized an important trigger for Joey was when he was tired and when they would raise their voices at him. Consequently, we encouraged them to avoid giving Joey too many demands when he was tired. With regard to raising their voices, we learned that particularly his father would yell when Joey was being uncooperative. His yelling led to some of the tension between Joey's parents regarding how discipline was being managed. To help resolve this conflict, we encouraged Joey's parents to become "robot disciplinarians," meaning that they were to give Joey consequences for uncooperative behavior in a "frank and matter of fact voice." We helped them recognize that when they yelled, Joey was actually more likely to be uncooperative.

\section{Termination Session}

At the final session, a posttreatment evaluation was conducted and although Joey's parents still rated him as being inattentive and aggressive on the CBCL, the overall elevation of their concerns decreased. In addition, on the PSI, both parents endorsed feeling competent regarding their ability to parent and Joey's mother felt much more supported by her spouse and less isolated. The DPIC observation revealed that Joey was complying to the first parental commands approximately $80 \%$ of the time and compliance was $100 \%$ when given a warning. Joey's mother gave frequent praises throughout the interaction. The session was best characterized by cooperation and laughter. Both Joey and his parents reported stronger positive feelings toward one another. His parents also reported an improvement in their marital relationship.

\section{CONCLUSION}

Having a child with ADHD can present significant disruptions within a family system. Frequently, these children are the identified patient, however, interventions that support the entire family are frequently indicated. Although on the surface, PCIT is a behaviorally oriented treatment program, we believe what really produces positive outcomes are the improved relationships. Parents begin to enjoy their children, perhaps for the first time. Children begin to feel special and want to please their parents. We have used this program with numerous families of ADHD children and, although there will continue to be conflicts, most families have been pleased with their results. Although Joey's parents may need a booster session (in fact, we recommend one 3 to 6 months after termination), we believe that they have been given strategies to help Joey cope with his difficulties in a consistent and supportive manner.

\section{REFERENCES}

Abidin, R. R. (1995). Parenting Stress Index (3rd ed.). Odessa, FL: Psychological Assessment Resources.

Achenbach, T. M., \& Endelbrock, C. S. (1983). Manual for the Child Behavior Profile and Child Behavior Checklist. Burlington, VT: Author.

Barkley, R. A. (1998). Attention-deficit hyperactivity disorder (2nd ed.). New York: Guilford.

Cunningham, C. E., \& Barkley, R. A. (1979). The interactions of hyperactive and normal children with their mothers during free play and structured task. Child Development, 50, 217-224.

Dixon, E. B. (1995). Impact of adult ADD on the family. In K.G. Nadeau (Ed.), A comprehensive guide to attention deficit disorder in adults (pp. 236-259). New York: Brunner/Mazel.

Eyberg, S. M., \& Robinson, E. A. (1983). Dyadic Parent-Child Interaction Coding System: A manual. Psychological Documents, 13, Ms. No. 2582. San Rafael, CA: Social and Behavioral Sciences Documents.

Hembree-Kigin, T. L., \& McNeil, C. B. (1995). Parent-child interaction therapy. New York: Plenum.

Lahey, B. B., Piacentini, J., McBurnett, K., Stone, P., Hatdagen, S., \& Hynd, G. (1988). Psychopathology in the parents of children with conduct disorder and hyperactivity. Journal of the American Academy of Child and Adolescent Psychology, 27, 163-170.

Mash, E. J., \& Johnston, C. (1990). Determinants of parenting stress: Illustrations from families of hyperactive children and families of physically abused children. Journal of Clinical Child Psychology, 19, 313-328.

McGee, R., Partridge, F., Williams, S., \& Silva, P. A. (1991). A twelve-year follow-up of preschool hyperactive children. Journal of the American Academy of Child and Adolescent Psychiatry, 30, 224-232.

Brian D. Johnson is a licensed child psychologist who specializes in treating children with childhood behavior disorders. He is an associate professor in the Division of Professional Psychology at the University of Northern Colorado and maintains an active private practice.

Leslie C. Franklin is a former school counselor who worked extensively with troubled youth. She is currently completing her doctoral 
186 THE FAMILY JOURNAL: COUNSELING AND THERAPY FOR COUPLES AND FAMILIES / April 2000

training in counseling psychology at the University of Northern Colorado.

Kathryn Hall has extensive inpatient treatment experience working with troubled teenagers and their families. She is currently completing her doctoral training in school psychology at the University of Northern Colorado.
Loreto R. Prieto is an assistant professor in the Department of Educational Psychology at the University of Oklahoma. He is a licensed psychologist who specializes in multicultural counseling and the supervision of beginning therapists. 